\title{
Analysis of the Clean Development Mechanism in China: A Deduction of Key Success Factors Using a Comparison among Implemented Projects and the Adaption to the Paris Agreement
}

\author{
Sönke NISSEN and Guan-wei HUANG* \\ Sino-German School for Postgraduate Studies (CDHK), Tongji University, Siping Road 1239, \\ Shanghai 200092, China \\ ${ }^{*}$ Corresponding author
}

Keywords: Clean development mechanism, Paris agreement, Key success factors, China, Emission trading system.

\begin{abstract}
The reduction of greenhouse gas emissions is a key action for stabilizing the global temperature at a tolerable level for mankind. International cooperation rolled out several mechanisms for the mitigation of climate change. This paper analyses the Clean Development Mechanism in regard to its impact on the momentarily negotiations of Paris. China as the world largest developer of projects within the CDM possesses a significant importance for the mechanism's development. This paper will therefore address the development of the mechanism over time and the impact on the Chinese sustainability. It will review some key features of and primary problems and finally present some suggestions on the further utilization within the Paris Agreement.
\end{abstract}

\section{Introduction}

The emission of carbon dioxide is one of the key challenges in the present time due to the acceleration of climate change. Since mankind continues to produce $\mathrm{CO} 2$ emissions the global mean temperature continues to increase. Nowadays the $\mathrm{CO} 2$ concentration in the atmosphere has reached a level of above $400 \mathrm{ppm}$ featuring a further linear growth. To keep the temperature increase limited, global efforts oppose the greenhouse gas emissions by adopting global mitigation schemes and offering possibilities for emission compensation.

One such scheme is the Clean Development Mechanism (CDM) which is defined in the Kyoto Protocol of the United Nations Framework Convention on Climate Change and thereby it is part of the international cooperation opposing the climate change.

The main purpose of the CDM is the reduction of greenhouse gas emissions by financing emission mitigation projects. Since its introduction in 2006 the CDM funded around 7,700 projects and thereby contributed to emission reductions.

This paper summarizes the finding of the corresponding master thesis about the progress and the limitations of the CDM in China as well as its influence on the Chinese sustainability. Since China is the member with most projects in the $\mathrm{CDM}$ as well as the biggest $\mathrm{CO} 2$ emitter nowadays, the paper seeks to answer questions about the performance of the mechanism, its key success factors and its implications on the Paris Agreement.

\section{Theoretical Background}

The CDM is a mechanism defined in the Kyoto Protocol (KP), which is a major instrument of the United Nations Framework Convention on Climate Change (UNFCCC) to oppose climate Change. The UNFCCC was adopted in 1992 and aims to stabilize of greenhouse gas concentrations in the atmosphere at a level that prevents dangerous anthropogenic interference with the climate system.

The KP as a supranational treaty and as a major instrument of the UNFCCC was adopted in 1997 and has the objective to reduce greenhouse gas emissions by committing parties of the UNFCCC to binding targets. The Convention in this regard distinguish between the developed Annex I and 
developing Non-Annex I parties and commits every Annex I parties to certain quantified emission limitation or reduction commitment (QELRC). In total, all QELRC result in an emission reduction of 18 percent until 2020 compared to 1990 .

The CDM is a flexible, market-based mechanism which encourages the realization of emission reduction projects. The CDM is constituted in Article 12 of the KP and defines the interchange of emissions budgets between Annex I parties and Non-Annex I parties without any QELRCs. These standardized emissions offset, namely certified emission reduction (CER), arise from projects in the developing country and equal one ton of $\mathrm{CO} 2$ equivalent, which can be traded in emission trading systems (ETS) in order to help Annex I parties fulfilling their QELRC.

Such, the CDM incorporates two main objectives. First, to enable developed countries with the compliance of their emission reduction commitments and secondly, to initiate and foster sustainable development in hosting, developing countries.

By obtaining these reductions in a developing country, the investing party typically can reduce its costs compared to the same reduction within its country. The developing country will benefit from the given possibility of low fiscal expenses to enhance sustainable investments in its economy and environment. This form of inward investments also encourages technology and knowledge transfer.

The projects realized within the CDM are always a joint action of two parties. In the hosting country, the project is realized with the financial and technical aid of the investing Annex I party. A baseline concept is used as a reference in the comparison to a project scenario in order to deduce emission differences and possible impacts of the project and thereby the baseline is crucial for the measurability of the project.

The project scenario implicates that the measures of the project activities result in reduced emissions compared to the baseline scenario. The resulting certified emission reduction is acquired to the assigned amount of the investing Annex I party

\section{Methodology}

This research uses several approaches to evaluate the CDM in China, compare Figure 1. First it conducts literature research to deduct the status quo before analyzing the impact of specific projects. As a consequence of the twofold objective of the CDM, the impact assessment is divided into two sub-objectives. The first objective - the emerged CER's - will be assessed through a comparison of target and the real emission reductions.

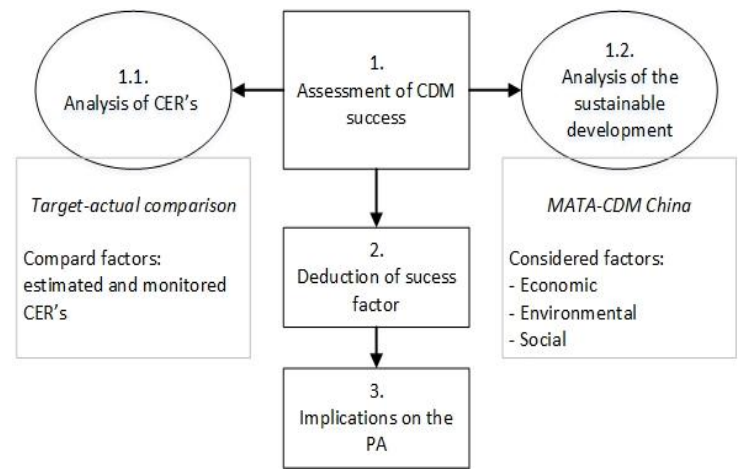

Figure 1. Assessment model of the thesis

The assessment of the sustainable development as second objective adopted the multi-attribute utility theory, namely the Multi-Attributive Assessment of CDM specific for China (MATA-CDM-China). 
Table 1. Assessment indicator system of MATA-CDM-China

\begin{tabular}{|c|c|c|c|c|}
\hline Type & Weight & Sub-Criteria & Weight & Type \\
\hline \multirow{3}{*}{$\begin{array}{l}\text { Social } \\
\text { Progress }\end{array}$} & \multirow{3}{*}{0.25} & Capacity development & 0.33 & Qualitative \\
\hline & & $\begin{array}{c}\text { Equal distribution of project } \\
\text { return }\end{array}$ & 0.37 & Semi-qualitative \\
\hline & & Employment generation & 0.3 & Semi-qualitative \\
\hline \multirow{3}{*}{$\begin{array}{l}\text { Environmental } \\
\text { protection }\end{array}$} & \multirow{3}{*}{0.43} & Air quality & 0.37 & Semi-qualitative \\
\hline & & Water \& Land quality & 0.29 & Semi-qualitative \\
\hline & & Fossil energy resources & 0.34 & Quantitative \\
\hline \multirow{3}{*}{$\begin{array}{c}\text { Economic } \\
\text { development }\end{array}$} & \multirow{3}{*}{0.32} & Sustainable technology transfer & 0.38 & Qualitative \\
\hline & & Regional economy & 0.26 & Semi-qualitative \\
\hline & & Micro-economic efficiency & 0.36 & Quantitative \\
\hline
\end{tabular}

This approach was significantly shaped by Sutter and modified for a Chinese specific usage by Zhang and Chen. It aims to represent the influence of CDM projects on China's sustainable development directly by an organic treatment of quantitative and qualitative information. The application of MATA-CDM-China implies three major characteristics:

First, MATA-CDM evaluates the impact of a project by one single overall utility, namely the sustainable development impact level (U). The utility $U$ ranges from -1 to 1 . A positive utility indicates a net positive effect on the sustainable compared to its baseline scenario. The more the utility differs from zero, the stronger is the project's effect on the sustainability. The utility U thereby enables the comparison between project activities regarding the sustainable development.

Second, MATA-CDM implies the weighting of sustainability criteria adjusted to the country of assessment. For this research the three sustainability criteria economic development, social progress and environmental protection with three sub-criteria each are shown in Table 1.

Third, MATA-CDM distinguishes between single and total utility values. The single utility value (u) is generated by a calculation of a specific utility function which describes the characteristic of sub-criteria. The total utility $(\mathrm{U})$ is calculated by weighting and accumulating all single utility values.

In this regard, it is necessary to explain that the core formula of MATA-CDM-China assessment method could be:

$$
U(P)=\sum_{i=1}^{n} w_{i} u_{i}\left[c_{i}(P)\right]
$$

In the formula above, $\mathrm{P}$ represents the individual project, $\mathrm{U}$ is the total utility, $\mathrm{w}_{\mathrm{i}}$ is the indicator weight, $\mu_{\mathrm{i}}$ the utility of indicator I, ci the sustainability of I

Additionally, several interviews serve as basis for the deduction of theories which are compared to the findings of the MATA-CDM-China.

\section{The CDM in China}

The assessment of the CDM shows a clear dominance of China within the mechanism. With more than 3700 projects China aggregated nearly 50 percent of all projects which account for about 60 percent of all reductions arising from the CDM. India as second largest member just accounts for roughly 11 percent.

Of the Chinese projects the majority with about 80 percent are large scale projects which are primarily located in the sectoral scope of energy industries.

Regarding the geographic distribution, CDM projects can be divided into three different areas of China, namely the western, central and eastern China with 369 projects, most projects are located in Sichuan closely followed by Yunnan with 368 and Inner Mongolia with 355 projects. Together with Gansu, these four provinces account for 35 percent of all projects in China. The reasons for this 
agglomeration of projects are the environmental conditions for hydropower plants in Sichuan and Yunnan as well as the conditions for wind power in Inner Mongolia and Gansu. Thereby these two renewable energy sources are the most frequent used ones in the Chinese CDM profile.

\section{Assessment of CDM Projects in China}

\section{Emission Reduction}

The total compliance rate of Chinese projects with their emission reduction target amounts to 78 percent. Thus, the first objective the reduction of greenhouse gas emission by the emerging CER's is fairly fulfilled. Nevertheless, it can be seen that the majority of projects cannot meet their estimated reduction target. About 45 percent of all projects reach less than 80 percent. Just a minority of 10 percent of all projects managed to fulfil their estimation or to exceed it. The degree of fulfilment ranges from 12 percent to 153.7 percent but pile up in the section around 80 percent target fulfilment.

\section{Sustainable Development}

The research confirms an overall successful implementation of the CDM in China regarding the sustainable development. Nearly all projects have a positive sustainability impact and with an average total utility around 0.37 the MATA-CDM-China verifies successes in the sustainable development and supports the theory of an overall positive effect of CDM projects in China. Furthermore, the research shows, that the economic development is one of the strongest aspects but being second to the environmental protection. Thus, the evaluation shows a clear trend towards environmental as the strongest and social as the weakest aspect.

The environmental and economic aspects account for approximately 35 percent of the total mean utility each. The social progress just contributes of 28 percent.

Environmental Protection. The environmental protection is the most important of all sustainability aspects by having a share of the average total utility 0,37 of about 0,14 . The great impact in this criterion is mainly due to the positive impact of projects on the air quality which features the highest utility in the assessment. On the other hand, the quality of water and land resources has the smallest utility in the study. Since most projects are renewable energy projects the research indicates a medium to major success of CDM project in the mitigation of the utilization of fossil energy resources. But in this regard the utility is below average and thereby the impact is lower than expected.

Economic Development. The economic development is the second most important aspects of the sustainability and nearly equals the utility of the environmental aspect with a share of 0,13 of the total utility of 0.37 .

The importance of the economic development is based on the regional economy. According to the study, the projects benefits the region, positively affect the life quality of residents independent of the project size and thereby enhance the economic development making it the second most important aspect. The microeconomic efficiency is among average and its importance minor compared to further aspects. The utility of the sustainable technology transfer is average as well and thereby reinforce the theory that it had high importance in the beginning but it is losing its relevance since China adapted rapidly to transferred technologies of developed countries.

Social Progress. With a contribution of merely 0,1 to the total utility 0,37 , the social progress is the weakest criterion in the Chinese sustainable development of Chinese CDM projects.

The capacity building has a high utility above average and emphasizes a positive effect on the building of organizational structures and sustainable economic activities. The utility of employment generation is the second lowest throughout the research featuring a small impact on job creation of the CDM in China. The strongest utility of the social progress is the equal distribution of project returns. 


\section{Summary}

Overall it can be said the CDM is an important mechanism and had major impacts on the development of a clean industry and environment in China.

The research indicates a compliance rate of Chinese projects with their estimated emission reduction target of 78 percent in average. Most projects possess a compliance rate between 70 and 100 percent. Consequently, this accomplishment can be regarded as a success of the CDM in China but leaves room for improvement especially since generation of CERs is the major objective of the CDM.

The sustainable development as the second objective of the CDM highlights successes as well. The average total utility has a value of 0.37 . The environmental protection and economic development nearly equal each other in the generation of major impacts. Social progress is the least successful aspect of the sustainability.

Major contributions by the CDM are the improvement of GHG emission reduction causing an improvement of the air quality and an enhancement of the regional economy by increasing the quality of life for residents and stakeholders. The least important aspects according to the case study are the generation of employment and the quality of land and water resources. Nevertheless, compared to the baseline scenario, all criteria feature a positive impact on the sustainability resembling a remarkable success of the CDM, but it likewise leaves room for improvement.

Since CDM activity has come to a near standstill and the overall sentiment about its recovery is widely pessimistic, a continuation of the CDM within the Paris Agreement is questionable. Unused potential will very likely be acquired by the CCER, a Chinese double mechanism of the CDM which will be enhanced in the following years.

From a Chinese point of view the CDM is a very successful mechanism which can benefit to the sustainable development of a county and help to reduce greenhouse gas emissions via the realization of projects. A possible approach of a future utilization could be the additionality of reductions supplementary to the national determined contribution in contrast to just the compliance with it.

\section{References}

[1] KOH, K. L.: Crucial issues in climate change and the Kyoto Protocol, 2010

[2] MASSAI, L.: The Kyoto Protocol in the EU, 2011

[3] MIAH, M. D.: Forests to climate change mitigation, 2011

[4] NDRC: CDM statistics, 2011

[5] NDRC: China certified emission reduction exchange info-platform, 2016

[6] SUTTER, C.: Sustainability assessment o energy related projects under the CDM of the Kyoto Protocol, 2003

[7] UNFCCC: The UNFCCC and its Kyoto Protocol, 2013

[8] UNFCCC: Introduction to the Convention, 2014

[9] UNITED NATIONS: United Nations framework convention on climate change, 1992

[10] UNITED NATIONS: Kyoto Protocol to the United Nations framework convention on climate change, 1998

[11] ZHANG, B.; CHEN, H.: Research on assessment of CDM projects' influence on China's sustainable development, 2010 